\title{
Puissance du dessin et limites du papier
}

Parole et pratiques graphiques inuit

\section{Aurélie Maire}

\section{(2) OpenEdition}

Journals

Édition électronique

URL : https://journals.openedition.org/clo/923

DOI : $10.4000 /$ clo. 923

ISSN : 2266-1816

Éditeur

INALCO

Édition imprimée

Date de publication : 15 janvier 2010

Pagination : 121-151

ISBN : 978-2-85831-196-5

ISSN : 0396-891X

Référence électronique

Aurélie Maire, "Puissance du dessin et limites du papier », Cahiers de littérature orale [En ligne], 67-68 2010, mis en ligne le 02 mars 2013, consulté le 01 juillet 2021. URL : http://journals.openedition.org/ clo/923 ; DOI : https://doi.org/10.4000/clo.923

Ce document a été généré automatiquement le 1 juillet 2021.

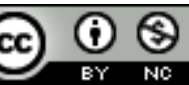

Cahiers de littérature orale est mis à disposition selon les termes de la Licence Creative Commons Attribution - Pas d'Utilisation Commerciale 4.0 International. 


\title{
Puissance du dessin et limites du papier
}

Parole et pratiques graphiques inuit

\author{
Aurélie Maire
}

J'aimerais que les gens pensent sérieusement à

l'art et y consacrent

tous leurs efforts. [...] Être un artiste est une

chose sérieuse.

Tiivi Ittuq ${ }^{1}$

1 La revalorisation de la langue et des savoirs revêt un caractère prioritaire pour les Inuit et leurs gouvernements, dans des sociétés où la langue représente un élément fondamental d'unité, mis à mal par le passé (politique fédérale "d'assimilation » et relocalisations forcées, notamment). Les créations artistiques contemporaines (sculptures, dessins, estampes, peintures, etc.) diffusées sur le marché international de l'art depuis $1949^{2}$ s'inscrivent dans cette dynamique culturelle. Mais quelle place accorde-t-on à l'inuktitut (langue inuit) et à l'oralité dans le domaine artistique inuit? Cet article amorce une réflexion liée à un double questionnement : sur ce que signifie «parler» et ce que signifie "dessiner», en considérant ces deux pratiques comme significatives dans l'élaboration des discours identitaires au Nunavut (arctique canadien). Qu'est-ce qui dans l'ordre de la parole renvoie aux images? Et inversement, qu'est-ce qui dans le trait dessiné sur le papier agit comme une parole? Les objets d'art sont-ils reconnus comme détenteurs d'une certaine forme de pouvoir à l'égal des mots? Ces questions nous conduisent ainsi à examiner les liens entre les notions de parole et de dessin, et plus largement entre l'oralité et les pratiques artistiques ${ }^{3}$.

2 Pour ce faire, j'appuie ma démonstration sur l'hypothèse selon laquelle l'oralité se définit comme un principe inhérent de l'art contemporain inuit et du dessin en particulier. Aux dires de nombreux Inuit, parmi lesquels compte l'artiste Pauloosie Kasadluak: «L'art nous aide à demeurer en lien avec notre culture » (Laugrand et al., 2003, 1). Il s'agit de tenter de déterminer la nature de ce lien, afin d'en saisir toute l'importance. La langue est ainsi souvent considérée comme indissociable de la culture. 
Pour T. Qumaq, auteur d'une encyclopédie publiée en inuktitut, la langue et la culture constituent le fondement de la communication sociale: «Les Inuit utilisent leurs coutumes et leurs mots en pouvant bien se comprendre et en pouvant discuter de ce qu'ils veulent et de ce qu'ils ne veulent pas, à n'importe quel sujet " (cité in Dorais, 1996, 274). Pour beaucoup, les pratiques artistiques contemporaines s'inscrivent dans le corpus des «savoirs anciens ayant encore de la pertinence aujourd'hui» (traduction littérale de qaujimajatuqait au Nunavut et piusituqait au Nunavik) dont le concept mobilise l'attention des gouvernements (Oosten et Laugrand, 2002).

Dans les sociétés inuit actuelles, la tradition orale conserve la préséance, ce dont attestent des recherches récentes en sciences sociales (Dorais, 1996; Therrien, 2002 ; Mahieu et Tersis, 2009). Le recours à la graphie ${ }^{4}$ acquiert cependant davantage d'importance, dans un contexte où l'émergence d'une conscience historique collective s'effectue en lien avec l'intégration d'éléments exogènes. Le besoin d'enregistrer les savoirs sur le papier se fait ainsi davantage ressentir, comme le souligne le témoignage d'Akisu Joamie :

Il faut que nous mettions sur papier notre savoir, même si jadis il n'était transmis qu'oralement. On évoque de plus en plus le temps où les Inuit étaient autonomes, où les familles étaient soudées et où la vie était agréable, et on parle de ce qui créait cette harmonie. Les Qallunaat [non-inuit] possèdent beaucoup d'informations écrites sur leur histoire et nous, les Inuit, nous devons commencer à écrire la nôtre pour que notre savoir se transmette et ne soit pas oublié.

(Therrien et Laugrand, 2001, 5)

4 Parmi les sources ethnographiques, celles de L. Turner (1894, 207-208), F. Boas (1888, 456-457), G. F. Lyon (1824, 294-295), K. Rasmussen (1931, 250 et 391-394) et K. BirketSmith $(1945,45-46$ et 161$)$ attestent notamment de l'importance des pratiques graphiques, dans les sociétés inuit de l'Alaska, du Groenland et du Canada, sous la forme de tatouages et d'incisions sur ivoire qui étaient parfois associés à des chants et des paroles "magiques». Au regard des sources littéraires disponibles en anthropologie comme en histoire de l'art, l'analyse des pratiques artistiques contemporaines inuit en relation avec la notion d'oralité ${ }^{5}$ reste en marge des travaux récents ${ }^{6}$. En anthropologie, la notion d'oralité est presque toujours associée à celle de «tradition orale », en référence à la cosmogénèse et aux savoirs des sociétés étudiées, transmis oralement (Goody, 2007, 51 ; Trudel, 2002 ; Laugrand, 2002).

L'oralité étudiée en lien avec la création artistique contemporaine réfute l'idée selon laquelle l'art serait un langage universel compte tenu de la spécificité de son statut « quasi magique » (expression de Kananginak Pootoogook cité in WBEC, 1979, 9) et de la valeur accordée à l'implicite et aux non-dits. L'historien d'art, T. Adorno, considère ainsi l'art comme un langage énigmatique : « Toutes les œuvres d'art et l'art en général sont des énigmes. Le fait que les œuvres disent quelque chose et en même temps le cachent place le caractère énigmatique sous l'aspect du langage » (Adorno, 1989, 159). La question du statut des images intervient parallèlement (Severi, 2003 ; Belting, 2004 ; Morphy, 2007) dans la lignée des travaux menés sur la perspective intentionnaliste (agency) développée par certains auteurs (Freedberg, 1991; Schaeffer, 1996 ; Bakewell, 1998 ; Gell, 1998). P. Descola s'inscrit dans la continuité de ces recherches tout en s'en distinguant puisqu'il favorise l'opération de figuration et s'intéresse aux représentations à "caractère iconique ", pour lesquelles il définit quatre formules ontologiques : animisme, totémisme, naturalisme, analogisme $(2006 ; 2009 ; 2010)$. Selon lui, figurer une ontologie propre à l'animisme « devrait consister à rendre visible 
l'intériorité des différentes sortes d'existants et à montrer que cette intériorité se loge dans des corps aux apparences fort diverses » (Descola, 2006, 173)7.

Notre démarche s'inscrit dans la lignée de ces travaux et s'appuie sur les résultats des analyses préliminaires des entrevues menées auprès d'une quarantaine d'acteurs culturels inuit et non-inuit au Nunavut, à Panniqtuuq, Kinngait et Iqaluit (ill. 1) ainsi que dans les institutions culturelles du Québec et de l'Ontario ${ }^{8}$. À ces éléments s'ajoutent des données linguistiques et iconographiques collectées sur le terrain, ainsi que des sources ethnographiques et archivistiques. Notons que les sources et les références iconographiques puisées presque exclusivement dans la tradition orale seront à peine évoquées ici (notamment Annaqtuusi, 1986 ; Blodgett, 1993 ; Eber, 1971 ; Routledge, 1990 ; Saladin d'Anglure et Amittu, 1978), au profit d'une approche qui place les notions d'«art» et d'«oralité" au centre la réflexion. Dans un premier temps, j'évoque brièvement le rôle social attribué aux pratiques artistiques par les Inuit, avant d'examiner les configurations de pensée associées au concept d'" art " (sanannguagaq) et de "parole» (uqausiq) qui appartiennent à un même registre. J'analyse ensuite comment « dessiner » et «parler » rendent tous deux visible une pensée (isuma), tout en favorisant la communication entre les êtres vivants et les êtres invisibles (défunts et esprits). J'achève enfin cette démonstration par l'évocation de dessins jugés inappropriés, en raison des sujets représentés qui, selon certains informateurs, ne devraient pas être publiquement révélés.

Illustration 1

Les communautés inuit au Canada (Source : http://www.ccca.ca)

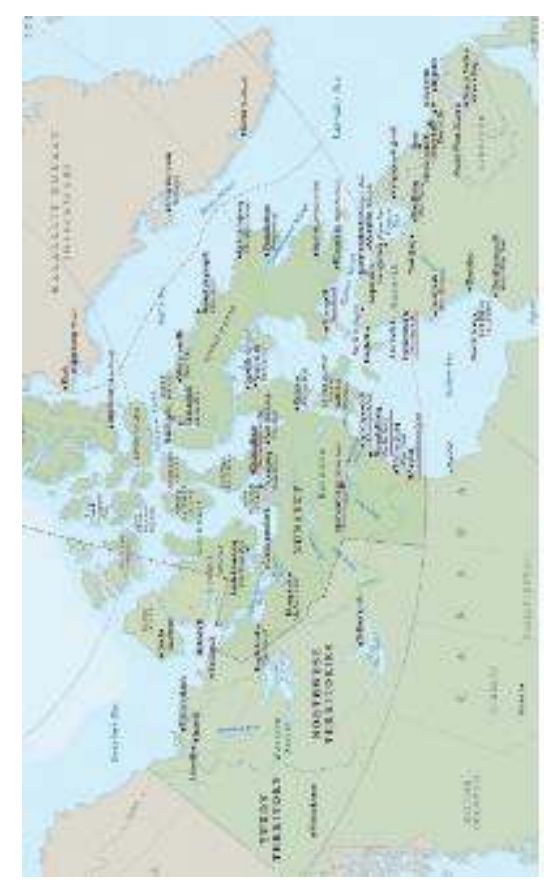

\section{L'art au quotidien}

Dans les sociétés occidentales où la dimension économique de l'art et la question des revenus des artistes relèvent souvent de l'ordre du non-dit, certaines déclarations d'auteurs inuit contredisent la théorie de l'« art pour l'art » développée par T. Gautier 
(1835), selon laquelle l'œuvre d'art se suffit à elle-même et est autonome. En dehors de toute conception élitiste de l'art, le pragmatisme des artistes inuit déroute parfois, dans un contexte où les activités artistiques représentent au Nunavut, la première source d'emploi non gouvernementale (Hill et Capriotti, 2009, 41). L'artiste Osuittuk Ipeelee souligne ainsi l'importance du développement de la création artistique à la fin des années 1940 :

Avant l'arrivée du gouvernement, il n'y avait qu'une seule façon de gagner de l'argent. Les gens pouvaient faire un peu d'argent, mais pas beaucoup avec un peu de fourrure de renard et d'autres choses qu'ils avaient l'habitude de rapporter. Ensuite, ils ont su que les sculptures faites ici dans l'Arctique avaient un prix et, quand le travail s'est mis à manquer, ils ont vite appris la valeur de ces objets. Voilà pourquoi ils ont consacré autant d'efforts à cette activité.

(Routledge, 1999, n. p.)

8 Le but initial du développement de l'art et de l'artisanat dans les territoires inuit canadiens visait effectivement, à la fin des années 1940, à procurer une nouvelle ressource économique pour remédier à certaines difficultés, comme la famine et les épidémies ${ }^{9}$. Si ces objectifs ont été atteints au-delà de toute espérance ${ }^{10}$, la production artistique génère aujourd'hui une certaine stabilité économique et sociale ${ }^{11}$. Mais ce succès n'en demeure pas moins relatif, au regard des conditions de travail des artistes dans les communautés inuit canadiennes, bien qu'il n'existe à ce sujet que peu de données. À Kinngait et Panniqtuuq, où je mène des recherches de terrain depuis 2006, de nombreux artistes admettent volontiers qu'ils arrêteraient de sculpter ou de dessiner s'ils pouvaient gagner davantage d'argent - cette situation semble récurrente au Nunavut et au Nunavik. En effet, vivre de l'art dans l'Arctique canadien reste une situation souvent précaire ${ }^{12}$ et il arrive fréquemment que des artistes cessent leurs activités artistiques au profit d'un emploi rémunéré dès que l'opportunité se présente. Compte tenu du manque d'offre d'emplois dans les communautés arctiques, de nombreuses personnes n'ont d'autres choix que de s'essayer à la sculpture et de vendre leurs pièces à la coopérative, afin de subvenir aux besoins de leur famille, alors que d'autres sculptent seulement pour compléter leurs revenus, ce que souligne l'ancien responsable de l'atelier d'estampe Dorset Fine Arts (propriété de la West Baffin Eskimo Cooperative) de Kinngait :

There is almost no other job in the community for some of these people and that [is why] they choose to try [carving] and create in an art form. Most of them are very successful, you know, and it is very important to support their family situation.

(Jimmy Manning, Kinngait 2007) ${ }^{13}$

Dans cette communauté, seulement une dizaine de dessinateurs et de maitres-graveurs sont employés par l'atelier local et reçoivent un salaire régulier (Maire, 2010, 34-35). Toutefois, si la création artistique contemporaine répond à des enjeux économiques individuels, familiaux et collectifs, elle dépasse cette seule finalité pour s'inscrire dans une démarche socioculturelle et politique, visant à la préservation et à la transmission de la langue et des savoirs. Bien que le développement de l'art inuit soit considéré comme le résultat d'une " acculturation » par certains anthropologues (Swinton, 1965; Graburn, 1969) et historiens d'art (Guigon et Le Marec, 2003; Pelaudeix, 2005), beaucoup d'Inuit conçoivent cette pratique en lien avec certaines coutumes funéraires et chamaniques. Le cas des miniatures réalisées dans l'os, l'ivoire ou le bois flotté, à des fins chamaniques en atteste (Laugrand et al., 2003 ; Descola, 2010, 53-60). Le témoignage de l'auteure Minnie Aodla Freeman rappelle ainsi qu'en dépit de l'appropriation de pratiques et de notions exogènes, l'art contemporain s'inscrit dans la continuité de la 
fabrication des petits objets et d'amulettes dont dépendaient autrefois le bien-être et la survie des Inuit :

Nous autres, Inuit, nous nous sommes adaptés et nous avons adopté bon nombre de mots pour décrire certains aspects de notre monde en mutation. Très souvent, nous forgeons un mot qui n'existe peut-être pas dans notre langue pour exprimer quelque chose d'une autre culture. Par exemple, il n'existait pas de mot pour dire « art » en inuktitut. Cela ne veut pas dire que l'art inuit n'existait pas, mais c'était une chose sérieuse, autrefois. Jadis, les Inuit faisaient des amulettes, des ornements pour le corps et du matériel de chasse, ainsi que des répliques d'objets de la vie courante qu'ils attachaient à leurs vêtements. Une grande partie de l'art traditionnel était exécuté pour des enterrements. Ces objets étaient pris au sérieux. Aux yeux des Qallunaat [non-inuit], l'emploi par les Inuit de certains charmes peut ne pas paraître très sérieux. L'art traditionnel servait en grande partie à chasser les mauvais esprits ou à porter bonheur lorsqu'un événement survenait, à encourager une jeune personne à être brave et aussi à conduire les morts vers les bons esprits, pour éviter que leur esprit ne flotte dans le néant. On confectionnait fréquemment un charme pour un nouveau-né. On en faisait aussi pour resserrer des liens particulièrement étroits. Certains de ces usages sont encore très courants aujourd'hui, en particulier le dernier. Ce n'est que lorsque les Qallunaat ont découvert cet art traditionnel que c'est devenu de l'« art ».

(Leroux et al., 1995, 14-15)

10 Autrefois, les Inuit conféraient aux objets fabriqués une fonction précise visant à maintenir un équilibre entre le monde des êtres vivants, celui des défunts et celui des esprits, selon le modèle d'une "ontologie animiste» telle que définie par P. Descola (2006). De façon plus pragmatique, la création artistique est considérée par de nombreux nunavummiut (habitants du Nunavut) tant comme un mode d'expression qu'une façon de vivre (Leroux et al., 1995 : 27) ; aussi, penchons-nous un instant sur les représentations conceptuelles inuit de la création artistique.

\section{L'œuvre, une réalité miniaturisée}

11 Bien qu'il y existe autant de définitions de l'art qu'il y a de cultures et d'individus, la dichotomie entre l'art et l'artisanat ${ }^{14}$ reste contraire aux modes de pensée amérindien et inuit. Au-delà de la définition de M. Mauss selon laquelle «un objet d'art, par définition, est l'objet reconnu comme tel par un groupe » (Mauss, 1926, 89), l'étude ethnolinguistique du concept d'« art » en inuktitut (sanannguaq) apporte de précieuses indications quant aux configurations de pensée qui s'y réfèrent. Suivant les travaux de N.Graburn (1969) et G. Swinton (1979), l'analyse morpho-sémantique indique que le syntagme sanannguaq se compose du lexème sana- «fabriqué, façonné » qui précède le morphème -nnguaq « réplique à échelle réduite ». En inuktitut, le terme sanannguaq (ou sanannguagaq), désigne ainsi toute production façonnée et manufacturée par l'homme, c'est-à-dire tout objet artistique ou artisanal et la sculpture, selon le contexte d'élocution. Il appartient ainsi au domaine du «fabriqué » (sana-) et inclut l'idée d'une reproduction miniature de la réalité (-nnguaq) ${ }^{15}$.

La pensée créatrice et esthétique inuit envisage la fabrication des objets selon le principe de réplique ou de copie de la réalité, selon un rapport d'échelle modifiée qui valorise davantage la capacité à "bien faire ", fondée sur la maîtrise technique et l'expérience du praticien. Dans cette perspective, l'apparence des personnes et des choses (tautu) importe peu, selon un système cosmologique où le pouvoir de 
transformation des êtres vivants et des objets nourrit les croyances et le répertoire iconographique des artistes (Poirier, 1992 ; Laugrand et al., 2000 ; Taylor, 2003 ; Perrin, 2010). Le témoignage d'une aînée, Tipuula Qaapik Atagutsiak, indique clairement que la facture prime sur l'apparence d'un objet ou d'une personne :

On m'a appris à ne pas juger une personne à partir de son apparence physique. On m'a conseillé de m'intéresser à ce qu'elle savait bien faire, par exemple confectionner des vêtements. Si j'appréciais la façon de travailler d'une femme, j'essayais de l'imiter. Dans le cas contraire, je m'abstenais. On m'a conseillé de ne pas porter de jugement et de ne pas dire qu'une personne est bonne ou mauvaise.

(Therrien et Laugrand, 2001, 124 et 109)

Les recherches de G. Swinton, menées à partir de concepts inuit vont en ce sens. Il rapporte: "The sananguaq concept tacitly entails a notion of reality in the sense that 'likeness' is achieved as 'replica of reality' - both actual and imagined - not beautiful but well made" (Swinton, 1979, 143) ${ }^{16}$. N. Graburn réfléchit quant à lui à idée de "réplique de la réalité » ou d'imitation qu'il met en relation avec la notion de "réalisme ", auparavant mentionnée, notamment par T. Mathiassen (1927, 120-122) dans une étude des motifs ornementaux incisés sur ivoire en Alaska, au Groenland et dans la région de Baffin. À partir de l'inuktitut, N. Graburn distingue deux concepts, en référence à ce système de valeur: sulijuq qui désigne "ce qui est véridique, vrai» relevant de l'ordre de l'expérimentation et takusurngnaituq qui correspond à une réplique imaginée (« imaginative replica») de ce qui n'a jamais été vu (Graburn, 1978, 72 ${ }^{17}$. Kenojuak Ashevak, illustre artiste de Kinngait ${ }^{18}$, précise ainsi : « I draw the thing I have never seen, the monsters and spirits, and I draw the old way, the things we did long ago before there were many white men » (Eber, 1971, 13). Lors des discussions, la majorité des artistes insistent sur l'importance de restituer la réalité passée et présente. Pauloosie Kasadluak explique ainsi le sens de sa démarche artistique :

Nous ne sculptons pas seulement pour l'argent. Pas plus que nous ne sculptons des choses inventées. Nous représentons la vie dans les temps anciens comme celle de maintenant. Nous montrons la vérité [...] Nous sculptons des animaux à cause de leur importance pour notre alimentation. Nous sculptons des personnages inuit parce que nous pouvons ainsi nous montrer au monde tels que nous étions autrefois et tels que nous sommes aujourd'hui [...] Nous sculptons pour montrer ce que nous avons réalisé en tant que peuple.

(Routledge, 1999, n. p.)

14 Pour presque tous les Inuit, la création artistique vise le partage des expériences, en favorisant la circulation de la parole, tant au sein des réseaux familiaux et communautaires qu'à l'extérieur des territoires inuit. C'est en ce sens que pour certains les pratiques artistiques jouent un rôle pédagogique majeur qui pourrait déterminer le futur des jeunes générations. Par exemple, Annie Manning, auteure et artiste de Kinngait, partage cet avis :

Les artistes de talent qui font des dessins et des sculptures peuvent montrer à nos enfants ce qu'était la vie; cela peut être utile pour leur avenir, les aider à faire quelque chose d'eux-mêmes.

(Leroux et al., 1995, 245) 


\section{La parole et le dessin ou les métamorphoses de la pensée}

Les nombreuses ethnographies consacrées aux sociétés inuit témoignent de l'importance accordée à la parole : celle-ci ne peut être prononcée sans que la véridicité du récit ne soit attestée par le locuteur et les auditeurs. Lors des discussions, la véracité des propos énoncés est en effet systématiquement rappelée : «Je vous dis seulement la vérité au sujet de ce que j'ai vécu. Je ne vous dirai rien concernant ce que je n'ai pas moi-même expérimenté. $»^{19}$ (Pitaloosie Saila, Kinngait 2009); ou encore: "Je ne parlerai pas de ce que j'ai entendu dire, parce que je ne l'ai pas vécu. Je parlerai uniquement de mes propres expériences. » (Kananginak Pootoogook, Kinngait 2010). Une parole est ainsi jugée fiable si le locuteur a été témoin de l'événement, mais également si une parole entendue provient d'une personne dont l'intégrité des propos ne peut être mise en doute ${ }^{20}$.

De même, lorsque les artistes sont interrogés sur leurs démarches artistiques et leurs intentions, ils rapportent souvent que leur inspiration prend source dans leur propre vécu: "I cannot draw anything that I myself did not experience ", explique Annie Putuguq (Kinngait 2009) (ill. 2). L'importance accordée à l'exactitude de ce qui est représenté, tant par les mots que par le dessin, rejoint la définition de uqaqtuq («il/elle parle ») proposée par T. Qumaq $(1991,101)$, dans son dictionnaire monolingue d'inuktitut : « Une personne, en disant qu'elle dit vrai, parce qu'elle ne dit que son idée, cette personne dit donc quelque chose de bon ou de mauvais ».

Illustration 2

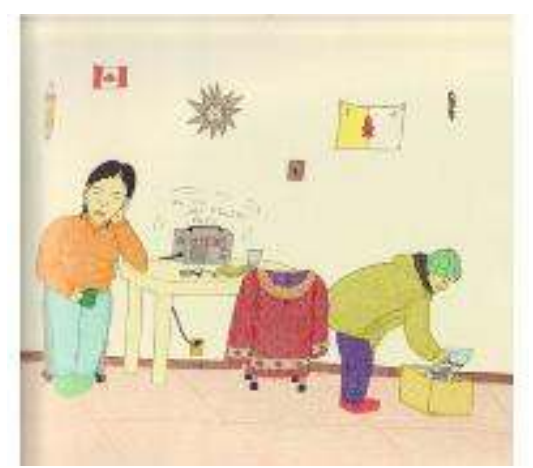

Annie Putuguq, 2005-2006, Calling Annie, encre et crayons de couleur sur papier. Collection privée, Toronto. (c) Reproduit avec l'autorisation de la Dorset Fine Arts

De ce point de vue, la parole détient une valeur morale puisque parler signifie « dire vrai » (suliniraqsuni : «cela s'avérant juste »). En parlant, nous sommes donc présumés dire la vérité, alors que le mensonge détourne la parole de son essence même. Parler, c'est faire partager son expérience et sa pensée, qu'elle soit estimée bonne ou mauvaise : «Les artistes dessinent ce qu'ils pensent... ils sont faits ainsi. Ils dessinent ce qu'ils pensent... et ce qu'ils ont vu " (Pudlo Pudlat cité in Routledge, 1990, 150). Dans cette perspective, créer une œuvre et parler répondent à la même exigence de " vérité ". À la question de savoir si la parole et le dessin tracé sur le papier peuvent être comparés, Pitaloosie Saila, dessinatrice expérimentée de Kinngait, répond immédiatement : 
Les histoires et les dessins inuit sont exactement les mêmes, ils parlent de la vie des Inuit du passé et d'aujourd'hui. Parler d'un sujet ou le dessiner est la même chose. Oui, je pense que les dessins et les mots ont un fort pouvoir.

(Kinngait 2010)

Si ce témoignage n'est pas isolé, tous les locuteurs ne sont cependant pas aussi explicites et s'expriment avec davantage de retenue, pour évoquer certains aspects du processus créatif. Aux dires de nombreux artistes, leur créativité résulte d'une démarche réflexive parfois difficile et tous insistent sur l'intervention de la pensée durant la genèse de l'œuvre comme lors de son exécution, tel Tiivi Ittuq :

Être un artiste est chose sérieuse. Un artiste peut travailler aussi régulièrement que n'importe quel autre salarié, mais il doit penser davantage. [...] Quand vous dessinez ou que vous faites des gravures, vous devez penser très fort.

(Weetaluktuk et Bryant, 2008, 9-100)

La parole comme la production artistique résulte effectivement d'une pensée que l'auteur exprime. Isuma désigne en inuktitut tant la pensée réflexive que la mémoire, les affects et la conscience. Dans son dictionnaire, T. Qumaq définit cette notion en termes d'immatérialité, de pouvoir, de mouvement et de sonorité (1991: 45), reprise par M. Therrien :

La pensée est par nature invisible et silencieuse; puissante, elle impulse un mouvement aux choses; elle déclenche des processus qui lui permettent de se manifester de façon tangible ; cette force infra verbale effectue un trajet de l'amont vers l'aval, de l'intérieur du corps (lieu de l'invisibilité et du silence) vers l'extérieur du corps où elle devient acoustiquement saisissable.

(Therrien, 2008, 275)

Par conséquent, l'objet d'art et la parole permettent de rendre les pensées (isumait) visibles et audibles, dans un mouvement tangible de l'intérieur vers l'extérieur du corps du sujet. Un dessin de Nikutai Saammajuali, une jeune artiste de Kinngait, a ainsi été réalisé pour les intervenants du service de santé local, afin d'expliquer aux patients le fonctionnement du cerveau (ill. 3). Dans cette représentation de la structure cérébrale et des capacités motrices et sensorielles du cerveau, apparaît la pensée, sous forme de points d'exclamation et d'interrogation. Ceux-ci sont représentés au crayon, par opposition aux autres éléments de la composition dessinés à l'encre noire, pour signifier le caractère latent et invisible de la pensée. Cette conception de la pensée repose sur la distinction de l'apparence physique et de l'intériorité (Descola, 2010). Selon cette définition, la pensée acquiert une dimension sonore qui lui est intrinsèque. Remarquons que certains artistes confèrent parfois au dessin une dimension acoustique. Par exemple à Qamanittuaq, Ruth Annaqtuusi Tulurialik désigne ses dessins par le terme qikaaluktut qu'elle définit comme "the sound of people passing by, perhaps outside your iglu, heard but not seen ». Selon elle, les dessins sont liés à une dimension sonore: "The stories and drawings are like sounds" (Annaqtuusi et al., 1986, 2). 


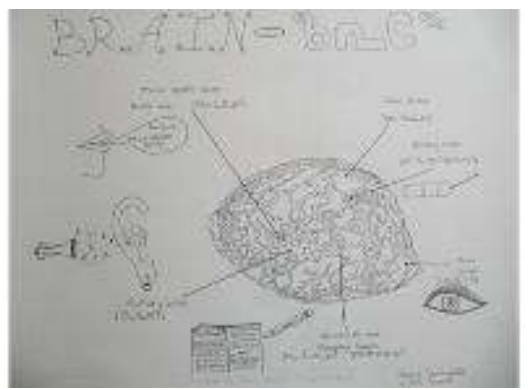

Nikutai Saammajuali, 2010, All about the brain - qaqitaup mitsaanuulingajuuinnait, encre et crayon noir sur papier, Dorset Fine Arts, Kinngait.

(c) Reproduit avec l'autorisation de la Dorset Fine Arts

\section{Illustration 4}

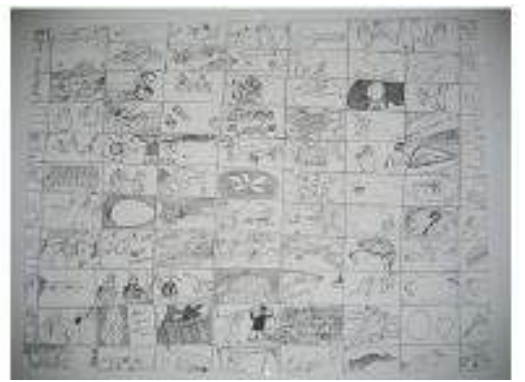

Suvinai Asuna, Sans titre, encre noire sur papier, Dorset Fine Arts, Kinngait.

(c) Reproduit avec l'autorisation de la Dorset Fine Arts.

Plus concrètement, les témoignages collectés associent systématiquement les représentations sculpturales, picturales et graphiques, au terme polysémique uqausiq qui désigne « un mot, une parole, un langage $»^{21}$. Bien qu'une représentation visuelle puisse « faire voir » (takutittivuq) la pensée de son auteur, une œuvre " dit » ou " parle » de quelque chose (uqaqpuq) davantage qu'elle ne «montre ». La représentation visuelle (tautunnguatauniq) comme la parole rend visible l'apparence des choses (tautu). Selon tous les artistes interrogés, les dessins appartiennent au corpus de la tradition orale puisque cette dernière nourrit leurs répertoires iconographiques et détermine la signification qu'ils attribuent aux œuvres. Il n'est pas anodin, par exemple, que des dessins puissent parfois être comparés à des pisiit (récemment appelées ajaaja), ces chants personnels que l'on composait autrefois pour exprimer le bonheur, les joies et les malheurs de la vie quotidienne. Jimmy Manning explique ainsi : «Les dessins et les pisiit sont identiques parce qu'ils racontent l'expérience des gens, ce que les familles ont vécu. » (Kinngait 2009). Quant à Qaunaq Mikkigak, une aînée respectée, elle ne conçoit pas le dessin sans le chant: "Je chantais toujours quand je dessinais.» (Kinngait 2009). Pour l'artiste Suvinai Asuna, les dessins représentent des fragments de la vie, d'expériences vécues dont l'exemple ci-dessous (ill. 4) est significatif : l'œuvre se compose près de soixante-dix rectangles juxtaposés contenant différents sujets. Elle explique ainsi, au sujet de ce dessin: "I was thinking of a big drawing but I ended up putting on paper different stories I had in my mind. Those are parts of my life." (Suvinai Asuna, Kinngait 2007). Les éléments de la composition du dessin apparaissent donc comme les composants du vécu de l'artiste. 


\section{Voir l'inaudible et entendre l'invisible}

Si parler et dessiner semblent appartenir au même ordre de représentation symbolique, quel pouvoir est-il attribué aux mots et à la parole (uqausiq)? La cosmogénèse inuit témoigne de la puissance des mots et de la parole. Les écrits de $\mathrm{K}$. Rasmussen indiquent qu'un chant magique fut à l'origine du genre féminin et de la sexualisation du monde. Alors que la terre avait engendré deux hommes qui voulurent enfanter après un certain temps de vie commune, ils entonnèrent ce chant : "Cet être humain, ce pénis, qu'il s'y forme un passage vaste et spacieux, passage, passage, passage !» (Rasmussen, 1929, 252). Le pénis de l'homme se fendit alors, il devint une femme qui donna naissance à un enfant. À cette époque, les mots avaient un pouvoir magique (irinaliutiit), ce dont témoigne cet autre mythe relatif à l'apparition de la clarté du jour. Dans les temps primordiaux, rapporte K. Rasmussen, la terre était plongée dans l'obscurité $(1929,253)$. Alors que le corbeau et le renard blanc comptaient parmi les premiers êtres vivants sur terre, ils se rencontrèrent et discutèrent ainsi :

«Qu'il n'y ait pas de jour, qu'il n'y ait pas de jour !» dit le renard, qui aime chasser dans l'obscurité. Mais le corbeau qui se déplace en volant et non pas en marchant, et qui se cognait constamment la tête - ce qui n'est pas étonnant -, se mit en colère et dit: « Qau ! Qau! Que la lumière surgisse, que le jour vienne ! - Taaq! Taaq! Qu'il fasse nuit, qu'il fasse nuit ! » répliqua le renard blanc. Depuis cette date, dit-on, il y a alternance du jour et de la nuit.

(Rasmussen, 1930, 26)

Ces deux exemples de récits empruntés à la cosmogénèse inuit constituent, en quelque sorte, les fondements mythiques d'un certain pouvoir attribué à la parole et semblent justifier son usage dans les pratiques chamaniques. Autrefois, les chamanes utilisaient parfois les mots comme des armes (sakkuit) contre leurs ennemis et une personne pouvait être tuée seulement par la parole (uqausinarmut), comme le mentionne $\mathrm{K}$. Rasmussen $(1931,201)$ : “Orpingalik [...] made evil magic words that were intended to kill". Une telle intention maléfique pouvait cependant être contrée par un chamane plus puissant qui renvoyait le maléfice à sa source. Jadis, la parole détenait un double pouvoir pouvant tout autant causer la mort ou la maladie que guérir une souffrance : "Même quand rien n'était dit, les angakkuit [chamanes] le savaient. Les angakkuit confrontaient la personne pour la faire avouer, mais si elle n'avouait pas, ça finissait par la tuer » (Saladin d'Anglure, 2001, 69).

Aujourd'hui, dans les communautés de l'Arctique canadien, le pouvoir des mots demeure tout aussi actuel, même si le chamanisme a été délaissé au profit du christianisme (Laugrand, 2002) : "Certains mots sont très puissants, très douloureux. Lorsque vous connaissez bien la signification de certains termes, vous vous rendez compte des douleurs qu'ils peuvent provoquer, même si ce ne sont que des mots " (Jaikku Pitseolak cité in Therrien et Laugrand, 2001, 305). La dualité de cette puissance reste très présente dans la pensée de la plupart des Inuit, même si les bienfaits de la parole sont aujourd'hui davantage valorisés, à des fins thérapeutiques (Pauktuutit, 1991). Il n'en demeure pas moins que parfois, des jeunes expriment leurs craintes quant au pouvoir des mots: "I'm afraid of old words" explique une jeune Inuk à une anthropologue qui lui demandait de l'assister comme interprète (Hervé, 2010, 15). De ce point de vue, la parole doit être parfaitement maîtrisée et retenue, car dans le cas contraire, le locuteur s'expose potentiellement à de graves conséquences ${ }^{22}$.

Cahiers de littérature orale, 67-68 | 2010 
La puissance conférée aux mots n'est pas sans évoquer le pouvoir de la pensée (isuma) définie, rappelons-le comme "invisible et silencieuse; puissante, elle impulse un mouvement aux choses [...]» (Qumaq, 1991, 45) et dont la parole est le produit. S'il semble difficile de comparer la puissance de la parole à celle de l'esprit, la force de la pensée mérite néanmoins d'être mentionnée, d'autant plus que les conséquences de pensées négatives ou maléfiques (isumanirluit) restent potentielles : « Si j’ai des pensées mauvaises ou négatives, je n'aurai pas une longue vie " (Lucassie Nutaraaluk cité in Korhonen, 2007, 4). La dissimulation peut également générer des effets sur la santé mentale et physique : "Si l'on refuse de divulguer un secret, on peut tomber malade " (Therrien et Laugrand, 2001, 164). L'impact de telles pensées ne semble néanmoins pas limité à la personne qui les produit, mais peut également générer des effets sur son entourage :

Même les pensées des gens pouvaient être perçues par les angakkuit. Si une personne avait caché quelque chose qui la tracassait, elle pouvait être la cause du problème affectant la communauté tout entière. [...] Les angakkuit étaient un instrument pour faire sortir les mauvaises pensées.

(Aupilaarjuk cité in Saladin d'Anglure, 2001, 75)

Il est parfois reconnu que « même après la mort, les pensées d'un aîné peuvent toujours se transformer en réalité " (Therrien et Laugrand, 2001, 308). De plus amples recherches mériteraient d'être engagées sur ce thème. Cette réflexion menée sur la notion de pouvoir de la parole et de la pensée vise à éclairer les configurations de pensée inuit auxquelles se réfère le dessin dont les témoignages insistent sur une relation inhérente entre l'art (sanannguagaq), la parole (uqausiq) et la pensée (isuma). Or, ces deux dernières étant considérées comme puissantes (sangijuqput: "ils/elles sont fort(e)s, puissant(e)s»), s'interroger sur le pouvoir potentiellement attribué aux pratiques du dessin (titirtugaq) et du tracé (taqsaq) ${ }^{23}$ apparaît pertinent.

\section{Pouvoir du tracé et puissance du dessin}

Les récits des origines du dessin et du marquage corporel apparaissent dans la cosmogénèse peu après l'avènement du jour et de la nuit, précédemment évoqué, bien qu'aucun pouvoir ne lui semble alors attribué. Selon le récit rapporté par Ivaluarjuk à K. Rasmussen $(1931,399)$, le corbeau était incolore à cette époque. L'histoire se poursuit ainsi :

Corbeau et huard, quand tous deux pouvaient prendre forme humaine, décidèrent un jour de se tatouer mutuellement. Corbeau fit de beaux tatouages sur le corps de huard. Mais quand ce fut son tour d'être tatoué, il trouva cela déplaisant et remua tellement que huard, fâché, lui renversa le récipient de suie sur tout le corps. C'est depuis ce jour que corbeau est noir.

(Saladin d'Anglure, 2006, 78-79)

Dans un autre récit cependant, celui d'un frère, Tarqiq, et d'une sœur, Siqiniq, qui deviendront par la suite la lune et le soleil, la trace et la marque sombres permettent de révéler plusieurs transgressions, dont celles de l'inceste et de la tromperie de Tarqiq qui abuse de sa sœur Siqiniq en dissimulant son identité, alors que la flamme de la lampe est éteinte ${ }^{24}$. Dans ce récit, la trace sombre ou la marque permet de rendre publiquement visible, de faire apparaître des transgressions et des secrets ${ }^{25}$. Cette exigence de visibilité qui reste très valorisée dans les sociétés inuit (Therrien, 2008, 276) confère ainsi à la trace, à la marque (taqsaq) un certain pouvoir ${ }^{26}$. Selon les données 
ethnographiques et archéologiques, une "intention magique » était parfois associée à ces pratiques graphiques, par l'intermédiaire des techniques de marquages corporels comme le tatouage et la peinture ou l'incision sur ivoire (Birket-Smith, 1936, 124 ; Rasmussen, 1931, 316). Aujourd'hui, des aînés, comme Aupilaarjuk, se remémorent le rôle protecteur de certains tatouages ${ }^{27}$ :

Si un ijiraq [êtres invisibles] essayait de s'approcher de moi, si j'avais un kigjugaq, un tatouage entre les yeux, cette marque agissait en tant que défense et me protégeait. L'jiiraq avait peur de cette marque. C'est comme ça que c'était à Nattilik.

(Saladin d'Anglure, 2001, 61)

Selon la formulation d'E.-M. Weyer (1932, 317-318), les tatouages peuvent être considérés comme des amulettes (aarnguat) qui conjurent les mauvais sorts et veillent au bien-être de leurs propriétaires. Si certaines marques corporelles exerçaient une fonction apotropaïque, un dessin ou une ligne pouvait également être tracée sur le corps à des fins thérapeutiques (Therrien et Laugrand, 2001, 78) ${ }^{28}$. Dans les dessins contemporains inuit, la représentation de tatouages, par les artistes expérimentés comme par les plus jeunes, ne se révèle donc pas anodine.

Établissant une analyse ethnolinguistique comparée entre « les traces dans la neige et les signes sur le papier ", M. Therrien montre comment la trace détient une puissance certaine de par son rôle véritable dans "l'unité des vivants et [...] l'interaction du visible et de l'invisible». Selon elle, la trace "est peut-être l'une des clés de la cohérence de la pensée, c'est-à-dire, un fait physique qui permet, par avancées successives, d'atteindre l'inobservable» (Therrien, 1990, 42). Cette notion de trace inhérente au dessin s'inscrit au cœur des relations des êtres humains aux forces invisibles ainsi que dans une démarche de visibilité qui reste étroitement liée à la parole. En ce sens et au regard des démarches artistiques entreprises par de nombreux Inuit, il n'est pas anodin d'entendre dire ainsi : "Drawing is healing to me" (Kinngait et Panniqtuuq, 2009 et 2010). Si la fonction curative de la pratique artistique est reconnue par les artistes comme par les professionnels de la santé, tous nos informateurs ont surtout souligné le sentiment de bien-être psychique (aanniaqtailimaniq) généré par la finalisation d'un dessin ${ }^{29}$. Mais une fois le dessin achevé, qu'en est-il de sa réception au sein de la communauté ? Bien que cette question reste en marge des études consacrées à l'art contemporain inuit, elle n'en demeure pas moins pertinente, car y répondre permettrait de mieux déterminer le pouvoir attribué aux images graphiques anciennes et récentes.

\section{Dessins « interdits »}

Le pouvoir souvent attribué aux mots et à la parole n'est pas sans évoquer la crainte et la méfiance suscitées à l'égard de certaines représentations comme celles des esprits (tuurngait). Autrefois, des réticences pouvaient être émises à l'encontre des Qallunaat (non-Inuit) qui, désireux d'acquérir des représentations de visions chamaniques ou des esprits, demandaient aux Inuit de les dessiner sur le papier. K. Rasmussen note ainsi que la figuration de l'esprit doit être cachée par crainte d'éventuelles représailles :

One day, I asked Anarqaq if he would try to draw me some of his spirit visions. He hesitated at first for fear offending the spirits, but when I promised him payment enough to include his helping spirits into the bargain, he agreed on the condition that I might do as I pleased with the drawings in the white man's country but 
undertake not to show them among his own people.

(Rasmussen, 1929, 44) réalisation des dessins d'esprits, considérée comme une transgression des règles (piqujaq : « ce qui est demandé, souhaité, ordonné, permis de faire »). Ce type de dessins a pourtant fait l'objet de collectes dans la région du sud Baffin, dès la fin du xIX ${ }^{e}$ siècle (Bloodget, 1993; Laugrand et al., 2000). Les objets gouvernés par des inuat, des forces tutélaires invisibles étaient également soumis à des interdits langagiers, (Rasmussen, $1929,214)^{30}$. Comme le rappelle Piona Keyuakjuk, un jeune artiste pluridisciplinaire, les savoirs chamaniques appartiennent au domaine du privé et les dessins qui s'y réfèrent ne doivent pas être publiquement révélés (Panniqtuuq, 2010). Il n'est d'ailleurs pas rare, aux dires de certains informateurs, que des dessins "confidentiels" (saqqititailiniit) soient conservés à l'abri des regards (en dépit de leur valeur monétaire potentielle). Les sujets en référence à la violence, l'alcoolisme, la souffrance ou la mort semblent appartenir à cette catégorie et sont, de ce fait, parfois jugés " politiquement incorrects » (katimaji pinillugaumatta), car ils transgressent des interdits (pittailiniit) et peuvent causer du tort à la communauté. «Tout le monde savait quand il se passait des drames dans les familles [...] on ne parlait pas de ces choses-là » explique-t-on (Panniqtuuq 2010). Est-ce commettre une "faute» (agliqtuq: "être soumis à un interdit ", aussi tirigusuktuq et tittailijuq) que de vouloir l'évoquer? Être considéré comme psychiquement souffrant et/ou physiquement malade impliquerait ainsi l'interdiction de fabriquer tout objet (Saladin d'Anglure, 2001, 105).

Selon Kenojuak Ashevak, rendre visible des actes violents ou représenter ses conséquences dans une création artistique n'a pas de valeur curative : «Il y a beaucoup de violence dans ces dessins, mais ces dessins-là n'aident pas les gens » (Kinngait 2009). À la vue de dessins jugés violents, des personnes détournent parfois le regard, refusant de s'exprimer à leur sujet. À l'inverse, d'autres considèrent que garder ces représentations visuelles dans la sphère domestique prive les plus jeunes de contreexemples, ce que souligne une mère de famille, travailleuse sociale à Iqaluit ayant souhaité conserver l'anonymat : « Nos enfants doivent savoir que ces choses-là existent, mais qu'elles ne sont pas normales. À la télévision, on sait que ces choses-là ne sont pas vraies. Le dessin dit la vérité, ce qui y est révélé est vrai» (Panniqtuuq 2010). Les créations artistiques permettent ainsi de «faire comprendre ce qui n'est pas agréable à entendre " (tukisinaqsitittiniq tusaruminanngittumik), dans un contexte où l'objet de pensée ne peut parfois être visualisé tant il reste émotionnellement douloureux (Oosten et Laugrand, 2007, 153). Un dessin de Jutai Toonoo, exposé en 2009 à la galerie Marion Scott à Vancouver, exprime par exemple le ressenti de la maladie (ill. 5). 


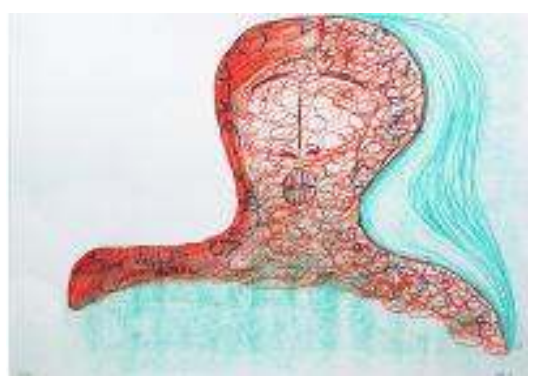

Jutai Toonoo, 2006-07, Continuous pain, crayons sur papier, collection privée.

(c) Reproduit avec l'autorisation de la Dorset Fine Arts

Il s'agit d'un portrait dont les pensées du sujet sont inscrites en inuktitut à l'intérieur de la ligne de contours qui forme le visage : “Sickness that we know, it hasn't stopped up to today, people who are close to me, they are tired, for one full month, I haven't walked, you can lose your mind" (traduction Marion Scott gallery). La bouche fermée du sujet témoigne de son incapacité à communiquer par la parole; les pensées restent donc silencieuses et invisibles (les yeux fermés révèlent cette impossibilité de voir). Selon plusieurs autres informateurs, la puissance du dessin ne fait aucun doute : «Les dessins sont très puissants. Ils disent davantage qu'ils ne laissent voir» (Piona Keyuakjuk, Panniqtuuq 2010).

L'attitude à adopter face au dessin et à la parole révèle parfois des contradictions qui oscillent entre l'exprimable et l'inexprimable, exposant le locuteur, dans les deux cas, à une position de vulnérabilité. "Silencieusement je parle, espérant être entendu ». Ce vers de l'un des poèmes d'Aalurtuq Aipili (Ipellie, 1975, 63), illustrateur, poète et auteur de talent originaire d'Iqaluit, rappelle que le silence (nipiqanngituq) et la parole ne sont pas contradictoires. Ceci n'est pas sans rappeler un proverbe yoruba (sud-ouest du Nigeria) selon lequel : "La bouche ne dit pas tout ce que les yeux voient " (cité in Laditan, 2004, 10). On apprend à bien parler, mais avant tout on apprend aussi à se taire. Une aînée tente de mettre ainsi fin à une discussion : «Ne fatiguons pas la seule bouche que nous avons pour en parler » (citée in Therrien, 2002, 118).

Cette analyse n'évoque que quelques-uns des aspects du rapport intrinsèque entre les pratiques graphiques et l'oralité dont la démonstration s'est structurée, dans une perspective ethnolinguistique et anthropologique, autour des représentations propres aux concepts de "parole-mot» et de "dessin-tracé ». Selon les configurations de pensée inuit, ces notions restent ainsi associées à une certaine forme de pouvoir. Jadis, la tâche du chamane avec l'aide de ses esprits auxiliaires visait à faire apparaître l'invisible, c'est-à-dire à révéler publiquement les manquements aux règles sociales et aux prescriptions rituelles, dont le but consistait à restaurer l'harmonie entre les êtres vivants et les entités invisibles. Dans cette perspective, le dessin apparaît comme une alternative puisqu'il permet de restituer la circulation de la parole, entre les générations, mais aussi entre les êtres vivants, qu'il s'agisse d'humains, d'animaux, de défunts ou d'esprits. Le dessin permet de révéler des pensées individuelles et collectives, tout en les véhiculant, selon un mouvement de l'intérieur vers l'extérieur $\mathrm{du}$ corps qui se positionne dans une dynamique relationnelle significative. Le choix des sujets iconographiques et la question de leur diffusion sur la scène publique suscitent cependant de vives controverses au sein des communautés inuit. Il n'en demeure pas moins que la pratique du dessin semble dotée d'un certain pouvoir transformateur qui 
agit sur la pensée et la parole, en donnant une apparence aux choses, une visibilité à l'invisible.

\section{BIBLIOGRAPHIE}

ADORno, Theodor, 1989, Théorie esthétique, Paris, Payot.

ANNAQtuUSI, Tulurialik Ruth, Pelly, David, 1986, Qikaaluktut Images of Inuit life, Toronto, Oxford University Press.

ARISTOTE, 1922, Poétique et Rhétorique, Paris, Librairie Garnier Frères.

BAKEWELL, Liza, 1998, Image acts, American Anthropologist, 100 (1), pp. 22-32.

Belting, Hans, 2004, Pour une anthropologie des images, Paris, Gallimard.

BIRKET-SMITH, Kaj, 1936, The Eskimos. Translated from the Danish by W. E. Calvert, London, Metheun.

BIRKET-SMITH, Kaj, 1945, Ethnographical Collections from the Northwest Passage. Report of the Fifth Thule Expedition, 1921-1924, Copenhagen, Gyldendal and New York, AMS Press.

BloDGETT, Jean, 1993, Strange Scenes: Early Cape Dorset Drawings, Kleinburg, McMichael Canadian Art Collection

BOAS, Franz, 1888, The Central Eskimo, 6th Annual Report, Washington and Lincoln, Smithsonian Institution-Bureau of Ethnology and University of Nebraska.

DESColA, Philippe, 2006, La fabrique des images, Anthropologie et Sociétés, 30 (3), p. 167-182.

Descola, Philippe, 2009, L'envers du visible : ontologie et iconologie, Histoire de l'art et anthropologie. Les procédures en histoire de l'art et en anthropologie, Paris, INHA/Musée du Quai Branly (« Les actes »), consulté sur Internet, http://actesbranly.revues.org, le 24 octobre 2009. DEsColA, Philippe, 2010, La fabrique des images. Visions du monde et formes de la représentation, Paris, Musée du Quai Branly.

DoRAIS, Louis-Jacques, 1996, La parole inuit. Langue, culture et société dans l'Arctique nord-américain, Paris, Peeters-Selaf.

EBER, Dorothy, 1971, Pitseolak: Pictures out of my life. From Interviews by Dorothy Harley Eber. With the Drawings and Prints of Pitseolak Ashoona / Pisiulak unikatuaq inuusiulauqtumik. apiqsinisuni nipiliarilauqtunga uma tasi igpu. Pisiula asuna titiqtugangit, Montréal, Design Collaborative Books. FREEDBERG, David, 1991, The Power of Images: Studies in the History and Theory of Response, Chicago, University of Chicago Press.

GAFFIOT, Félix, 1934, Dictionnaire latin français, Paris, Hachette.

GAUTHIER, Théophile, 1835, Mademoiselle de Maupin, Paris, Eugène Renduel.

GeLL, Alfred, 1998, Art and Agency. An Anthropological Theory, Oxford, Clarendon Press.

Goody, Jack, 2007, Pouvoirs et savoirs de l'écrit, Paris, La Dispute/Snédit. 
GOVERNMENT OF NUNAVUT, 2007, Sanaugait. A Strategy for Growth in Nunavut's Arts and Crafts Sector Sanaugait / Piruqpallianasuarniq nunavummi sanaugalirinirmit, Pangnirtung, Department of Economic Development \& Transportation, Community Economic Development Division.

GRABRUN, Nelson, 1969, Art and acculturative processes, International Social Science Journal, 21, pp. 457-68.

GRABRUN, Nelson, 1978, Understanding Contemporary Inuit art. Differing Values and Attitudes of Artists and collectors', Arts \& Culture of the North, 2 (1), pp. 71-73.

GuIGON, Gwénaèle, LE MAREC, Aurélie, 2003, Créativité et création, l'art contemporain inuit in M. Therrien (éd.), Les Inuit de l'Arctique canadien, Québec, CIDEF-AFI Faculté des Lettres, Université Laval, p. 131-146.

HILL, Kelly, CAPRIOTTI, Kathleen, 2009, Les artistes dans les provinces et territoires du Canada basé sur le recensement de 2006, in ministère du Patrimoine canadien et Conseil des Arts de l'Ontario, Conseil des Arts du Canada (éd.), Regards statistiques sur les arts, vol. 7, n 5, Ottawa, Hill Stratégies Recherche Inc.

IPELLIE, Alootook, 1975, [Untitled poetry], Inuit Today/Inuit Ullumi, 4 (7), p. 63.

HERVÉ, Caroline, 2010, Analyser la position sociale du chercheur : des obstacles sur le terrain à l'anthropologie réflexive, Les Cahiers du Ciéra, 6, p. 7-26.

KTA (éd.), 2008, Répercussions socioculturelles des industries culturelles autochtones. Document de travail, Toronto, KTA Centre for Collaborative Government.

KoRHONEn, Marja, 2007, Titatuuniq. Aniguatittiniq Pijuriatujunik ammalu Sivumuagiarniq Naggaittukkut / Resilience. Overcoming Challenges and Movingon Positively, Ottawa, Ajunnginiq Centre, National Aboriginal Health Organization (NAHO).

LADITAN, Affin O., 2004, De l'oralité à la littérature : métamorphoses de la parole chez les Yorubas, Semen, [De la culture orale à la production écrite : Littératures africaines], 18, p. 1-10.

LAUGRAND, Frédéric, 2002, Écrire pour prendre la parole. Conscience historique, mémoires d'aînés et régimes d'historicité au Nunavut, Anthropologie et Sociétés, 26 (2-3), p. 91-116.

LAUGRAND, Frédéric, Oosten, Jarich and TRUDel, François, 2000, Nunavummi iqqaumajaujullu qanuinnirijauvaksimajullu ilangit. naasautaa 1. Tuurngait tautunnguaqtauningit / Memory and History in Nunavu. Representing Tuurngait, vol. 1, Iqaluit, Silattuqsarvik Nunavut Arctic College.

LAUGRAND, Frédéric, OOSTEN, Jarich and TRUdel, François, 2003, Nunavummi iqqaumajaumajullu qanuinnirijausimajullu ilangit. Uppirniqainnrniq / Memory and History in Nunavut. Keeping the Faith, Iqaluit, Nunavut Arctic College.

Leroux, Odette, JACKSON, Marion and Audla FreEman, Minnie (eds), 1995, Inuit Women Artists, Voices from Cape Dorset, Hull, Canadian Museum of Civilizations.

LYON, George Francis, 1824, The Private Journal of Captain G. F. Lyon, of H.M.S. Hecla, During the Recent Voyage of Discovery under Captain Parry, 1821-1823, London, J. Murray.

MAHIEU, Marc-Antoine, Tersis, Nicole (eds), 2009, Variations on Polysynthesis. The Eskaleut languages, Paris, Université Paris 3-CNRS-CELIA.

MAIRE, Aurélie, 2010, La diffusion de l'art contemporain inuit canadien par Internet : de l'œuvre d'art à l'internaute, Les Cahiers du Ciéra, 5, p. 31-51. 
MATHIASSEN, Therkel, 1927, Archaeology of the Central Eskimos: The Thule Culture and Its Position Within the Eskimo Culture. Translated from the Danish by W. E Calvert. Report of the Fifth Thule Expedition, 1921-24, Copenhagen, Nordisk.

MAuss, Marcel, 1926, Manuel d'ethnographie, Paris, Éditions sociales.

MORPHY, Howard, 2007, Becoming Art. Exploring Cross-Cultural Categories, New York, British Library.

MYERS, Marybelle, 1984, A Brief History of the Marketing of Canadian Inuit Art, in Lipton Barbara (ed.), Arctic vision. Art of the Canadian Inuit, Ottawa, Canadian Arctic Producers, pp. 99-103.

OOSTEN, Jarich, LAUGRAND, Frédéric (eds), 1999, Interviewing Inuit Elders. Volume 1. Introduction. Iqaluit, Nunavut Arctic College.

OOSTEN, Jarich, LAUGRAND, Frédéric (eds), 2002, Qaujimajatuqangit and social problems in modern Inuit society. An elders workshop on angakkuuniq, Études Inuit Studies, 26 (1), pp. 17-44.

OOSTEN, Jarich, LAUGRAND, Frédéric (eds), 2007, Surviving in Different Worlds: Transferring Inuit Traditions from Elders to Youth, Iqaluit, Silatusarvik Nunavut Arctic College.

PAUKTUUTIT, Inuit Women's Association, 1991, No More Secret / Ijirartuaqarunniirniq, Ottawa, Pauktuutit Arnait Inuit katujiqatigiingit Inuit Women's Association Pauktuutit.

PelaudeIX, Cécile, 2005, Modèles de temporalité et lieux du sens en histoire de l'art : dessins et estampes (1959-2002) de l'artiste inuit Kenojuak Ashevak. Thèse de doctorat, Université Laval, Québec, et Université Pierre Mendès-France, Grenoble III.

Perrin, Michel, 2010, Voir les yeux fermés. Arts, chamanismes et thérapies, Paris, Seuil.

PEYTARD, Jean, 1970, Oral et scriptural : deux ordres de situations et de description linguistiques, Langue française, 6, p. 35-39.

PoIRIER, Sylvie, 1992, Cosmologie, personne et expression artistique dans le désert occidental australien, Anthropologie et Sociétés, 16 (1), p. 41-58.

QUMAQ, Taamusi, 1991, Inuit uqausillaringit. Les véritables mots Inuit : un dictionnaire des définitions en inuktitut du Nunavik, Québec arctique, Québec, Inukjuak, Montréal, Association Inuksiutiit Katimajiit \& Institut culturel Avataq.

QUMAQ, Taamusi, 2010, Je veux que les Inuit soient libres de nouveau. Autobiographie (1914-1993), Québec, Les Presses de l'Université du Québec.

Randa, Vladirmir, 2009, Uumajunik uqaruluujaqtuq “speaking badly to/about the animals" or how human speech affects them, in B. Colignon et M. Therrien (eds), Orality in the 21st century: Inuit discourse and practices. Proceedings of the 15th Inuit Studies Conference. Consulté sur Internet (http://www.inuitoralityconference.com/) le 5 janvier 2010.

RASMUSSEN, Knud, 1929, Intellectual Culture of the Iglulik Eskimos. Report of the Fifth Thule Expedition 1921-1924, Kobenhavn, Gyldendalske Boghandel, Nordisk Forlag.

RASMUSSEN, Knud, 1930, Iglulik and Caribou Eskimo Texts. Report of the Fifth Thule Expedition 1921-1924, Kobenhavn, Gyldendalske Boghandel, Nordisk Forlag.

RASMUSSEN, Knud, 1931, The Netsilik Eskimos: Social Life and Spiritual Culture. Report of the Fifth Thule Expedition, 1921-1924, Copenhague, Nordisk Forlag.

RoutleDge, Marie, 1990, Pudlo : Trente années de dessin, Ottawa, Musée des Beaux-Arts du Canada. 
ROUTLEDGE, Marie, 1999, Sanannguarnikkut inuunirmik. Inuit sanaugangit kanataup takujaksaqarvingannit / Un art qui s'affirme. La sculpture de la collection permanente, Ottawa, Musée des Beaux-Arts du Canada / National Gallery of Canada.

SALADIN D'ANGLURE, Bernard (éd.), 2001, Innarnik Apiqsuqattarniq. Naisautaa 4. Akpirijavinituqait Angakkuillu / Interviewing Inuit Elders. Volume 4. Cosmology and Shamanism / Entrevues avec des aînés inuit. Volume 4. La cosmologie et le chamanisme inuit, Iqaluit, Nunavut Arctique College.

SALADIN D’ANGLURE, Bernard (éd.), 2006, Être et renaître inuit homme, femme ou chamane, Paris, Gallimard.

SALADIN D'ANGLURE, Bernard, AMITTU, Davidialuk Alasuak, 1978, La parole changée en pierre, vie et œuvre de Davidialuk Alasuak, artiste inuit du Québec arctique, Québec, Gouvernement du Québec, ministère des Affaires culturelles, Direction générale du Patrimoine.

SEVERI, Carlo, 2003, Pour une anthropologie des images. Histoire de l'art, esthétique et anthropologie, L'Homme, 1 (165), p. 7-9.

SCHAEFFER, Jean-Marie, 1996, Les célibataires de l'art. Pour une esthétique sans mythes, Paris, Gallimard.

SwINTON, George, 1965, Eskimo sculpture / Sculpture esquimaude, Toronto, McClelland and Stewart. SWINTON, George, 1979, Contemporary Inuit Aesthetics: touch and the real, Arts \& Culture of the North, 3 (2), pp. 143-145.

TAYLOR, Anne-Christine, 2003, Les masques de la mémoire. Essai sur la fonction des peintures corporelles jivaro, L'Homme, 1 (165), p. 223-48.

THERRIEN, Michèle, 1990, Traces sur la neige, signes sur le papier. Significations de l'empreinte chez les Inuit Nunavimmiut (Arctique québécois), Journal de la Société des Américanistes, LXXVI, p. 33-53.

THERRIEN, Michèle, 2002, Ce que précise la langue inuit au sujet de la remémoration, Anthropologie et Sociétés, 26 (2-3), p. 117-37.

THERRIEN, Michèle, 2008, Tout révéler et rester discret chez les Inuit de l'Arctique canadien, in M. Therrien (éd.), Paroles interdites, Paris, Karthala-Langues'O, p. 251-284.

THERRIEN, Michèle, LAUGRAND, Frédéric (eds), 2001, Innarnik Apiqsuqattarniq. Aannianiq Aanniaqtailimaniq / Interviewing Inuit Elders. Perspectives on Traditional Health / Interviews d'Aînés Inuit. Bien-être Physique et Psychique, Iqaluit, Nunavut Arctic College.

TRUDEL François, 2002, De l'ethnohistoire et l'histoire orale à la mémoire sociale chez les Inuit du Nunavut, Anthropologie et Sociétés, 26 (2-3), p. 137-59.

TURNER, Lucien, 1894, Ethnography of the Ungava District, Hudson Bay Territory. In Eleventh Annual Report of the Bureau of American Ethnology for the Years 1889-1890, Washington, Government Printing Press.

WALK, Ansgar, 1999, Kenojuak: the life story of an Inuit artist, Manotick [Ontario], Penumbra Press.

WATT, V. J. (ed.), 1980, Canadian Guild of Crafts Quebec, the Permanent Collection Inuit Arts and Crafts C. 1900-1980, Montréal, Canadian Guild of Crafts Québec.

WBEC, West Baffin Eskimo Cooperative (ed.), 1979, Twentieth Annual Cape Dorset Graphics Collection. Dorset 1979, Toronto, M. F. Feheley Publishers. 
WEETALUKTUK, Jobie, BRYANT, Robyn, 2008, Tiivi Ittuup inuusinga sanannguarusingalu unikkausingillu / Le monde de Tivi Etok : la vie et l'art d'un aîné inuit de Kangiqsualujjuaq, Nunavik / The world of Tivi Etok: The life and art of an Inuit elder from Kangiqsualujjuaq, Nunavik, Québec, Éditions MultiMondes.

WEYER, Edward-Moffat Jr., 1932, The Eskimos: Their Environment and Folkways, New Haven, Yale University Press.

\section{NOTES}

1. Tiivi Ittuq (Teevee Etook) cité in Weetaluktuk et Bryant $(2008,99)$. Traduction de Siasie Iqaluk Longue Épée.

2. En 1949 eut lieu à la Guilde canadienne d'art et d'artisanat de Montréal la première exposition-vente de sculptures contemporaines inuit sur le marché international de l'art. Son vif succès auprès des critiques et amateurs d'art encouragea les artistes inuit à poursuivre leur entreprise (Watt, 1980 ; Myers, 1984).

3. Ces questions restent au centre d'une réflexion qui s'inscrit plus largement dans le cadre d'une recherche doctorale consacrée aux pratiques artistiques graphiques (dessin et techniques d'estampe) dans les sociétés inuit contemporaines du Nunavut. Celle-ci s'effectue en cotutelle à l'Inalco, section langues et cultures des Amériques, Centre d'étude et de recherche sur les littératures et les oralités du monde (Cerlom); et à l'université Laval, département d'anthropologie, Centre interuniversitaire d'études et de recherches autochtones (Ciéra). Depuis 2006, mon travail bénéficie du soutien financier de l'Inalco et de l'université Laval, ainsi que de l'Institut Polaire français Paul Émile Victor (IPEV, programme 1168), du Centre National de Recherche Scientifique (CNRS, GDR 3062 : « Mutations Polaires : sociétés et environnement »), du Conseil de Recherches en Sciences Humaines du Canada (projet CRSH « Transmission des savoirs, oralité et résilience »), du Programme de Formation scientifique dans le Nord (PFSN). Je remercie sincèrement ces institutions, ainsi que mes codirecteurs Michèle Therrien et Frédéric Laugrand, pour leur appui respectif. J'adresse également mes remerciements aux relecteurs de cet article pour la justesse de leurs commentaires et les discussions qui en résultent.

4. L'introduction de l'écriture sous forme de caractères syllabiques se situe à la fin du XIX $\mathrm{e}^{\mathrm{e} i e ̀ c l e}$, alors que les missionnaires moraves tentaient de diffuser la Bible dans les territoires inuit canadiens (Laugrand, 2002).

5. Selon J. Peytard (1970) et A.-O. Laditan (2004), l'oralité est le caractère des énoncés réalisés par articulation vocale, susceptibles d'être entendus. Cette conception de l'oralité prend en compte la parole comme un langage articulé, inséparable des caractéristiques qui l'entourent telles que la diction, l'intonation, le débit, les accents, les pauses, etc. Elle intègre également une situation d'échanges où émetteur et récepteur sont en contact direct (situation de face à face) ou indirect (isolés l'un de l'autre).

6. Deux publications font cependant date dans ce domaine: le catalogue de la première exposition monographique dédiée à un artiste inuit - Pitseolak Ashoona en l'occurrence - qui publie les entrevues menées en inuktitut avec l'artiste (Eber, 1971); et le catalogue consacré à Davidialuk Alasua Amittu qui explique ses œuvres par des récits issus de la tradition orale (Saladin d'Anglure et Amittu, 1978).

7. Dans la lignée de ces recherches, M. Perrin se consacre aux pratiques thérapeutiques ayant recours à des objets et des images utilisés par les praticiens pour communiquer avec le «mondeautre ", le monde invisible des entités non humaines (esprits, défunts, etc.), dans une perspective comparative entre les populations amérindiennes et celles de l'Angola, de l'éthiopie et de la Nouvelle Guinée (Perrin, 2010, 12). Ces travaux attestent d'un lien inhérent de ces pratiques à la parole et au chant. En Australie occidentale, S. Poirier interroge, quant à elle, le pouvoir 
d'évocation des représentations graphiques des sociétés aborigènes, selon une "valeur ontogénique et ontologique du lien qui unit la personne, l'image et le territoire » et dans laquelle l'oralité reste centrale (Poirier, 1992, 55).

8. Ont notamment participé à la recherche: des artistes et leur entourage familial, les responsables des ateliers d'art graphique et des coopératives locales à Kinngait et Panniqtuuq, les acteurs d'institutions culturelles inuit, des professionnels du marché de l'art autochtone et inuit. 9. Se référer notamment au récit autobiographique de Kenojuak Ashevak (Walk, 1999).

10. La première exposition-vente de 1949 à Montréal amorce ainsi la diffusion commerciale de la sculpture et de l'estampe inuit sur le marché international de l'art (Watt, 1980).

11. Les retombées économiques des activités artistiques permettent ainsi aux membres de la coopérative locale de diversifier les services et de développer les infrastructures: magasin général, bureau de poste, formations professionnelles, services bancaires, vérifications comptables, activités de tourisme et d'hôtellerie, constructions de bâtiments, distribution de produits pétroliers, pourvoiries de chasse et de pêche, etc. (Government of Nunavut, 2007 ; KTA, 2008, notamment).

12. Les statistiques de 2006 montrent qu'un artiste du Nunavut gagne tout juste un tiers du revenu moyen de tous les travailleurs du Nunavut, soit une moyenne de 26800 \$ par année (Hill et Capriotti, 2009, 41). 1,86\% de la population du Nunavut se déclare être un(e) artiste travaillant à mi-temps ou à temps plein, contre $0,77 \%$ au Canada (ibid, 7).

13. N. Graburn dresse un constat identique, alors qu'il travaille avec les sculpteurs de l'île de Baffin dès les années 1960 (1978, 72): "When I asked hundreds of Inuit about their attitude towards carving, the majority replied: 'I do it inurutiksatuariraku because it is the only way I have to make a living."”.

14. L'étymologie latine ars, artis dont le mot «art» est issu distingue trois sens: 1) talent, savoir-faire, habile ; 2) ce à quoi s'applique le talent, le savoir-faire ; métier, profession ; science ; 3) connaissance, technique, théorie, corps de doctrine, système (Gaffiot, 1934, 165). La polysémie du mot ars, artis induit l'idée que toute production résulte d'un savoir-faire, d'une habileté, d'une connaissance technique et/ou théorique : la créativité est donc inhérente à la maîtrise de ces savoirs. Au viII ${ }^{\mathrm{e}}$ siècle, on ne distingue alors pas les arts des sciences. À partir de la Renaissance italienne, c'est-àdire aux fondements mêmes de la discipline de l'histoire de l'art, s'élabore en Occident la distinction entre l'art et l'artisanat.

15. Le morphème -nnguaq peut ainsi être utilisé dans d'autres contextes d'élocution, par exemple celui du jeu: pinnguaq, "une réplique d'une chose»; une poupée inunnguaq "un homme en miniature ».

16. Ces propos rejoignent la pensée d'Aristote (Poétique, chapitre II), qui définit la mimésis comme l'art de représenter la réalité selon deux principes: la simple imitation de la nature et la stylisation de celle-ci.

17. Comme le mentionne G. Swinton $(1979,143)$, la terminologie à laquelle se réfère N. Graburn est celle du Nunavik (Arctique québécois) et ne correspond pas à l'ensemble des dialectes de l'Arctique canadien. En effet, lors de mes enquêtes menées à Panngiqtuuq et Kinngait, le terme isumannguaq (littéralement "une réplique miniature de la pensée ») est systématiquement employé pour désigner toute représentation issue de l'imagination ou tout sujet iconographique qui ne résulte ni de l'expérience individuelle et collective, ni de récits de la cosmogénèse inuit (unikkatuat).

18. Kenojuak Ashevak est la première artiste inuit à devenir membre de l'Ordre du Canada en 1982.

19. Dans cet article, tous les extraits des entretiens cités en français ont été traduits de l'inuktitut, par l'auteur. Les extraits qui apparaissent en anglais ont été formulés dans cette 
langue par les participants de l'enquête. Pour chaque extrait d'entretien est mentionné : le nom $\mathrm{du}$ locuteur, le lieu et l'année de l'enquête.

20. Ainsi, en inuktitut, la terminologie qui relève du processus de remémoration est déterminée par la participation du locuteur aux événements mentionnés, en tant qu'observateur ou en tant qu'acteur (Therrien, 2002).

21. T. Qumaq par exemple, définit uqausiq $(1991,100)$ : «La personne peut dire ce qu'elle veut dire n'importe comment, parce que sa bouche est mouillée, [elle peut énoncer] sa parole n'importe comment ». (cité in Dorais, 1996 : 266).

22. Dans des recherches consacrées à la relation entre le règne animal et les êtres humains, V. Randa (2009) montre ainsi que s'adresser de façon inappropriée au gibier en lui parlant «mal » (uqaruluujaqtuq : «Il/elle parle mal ») ou «trop» (uqausiuluartuq : «Il/elle parle trop ») perturbe l'équilibre relationnel entre les êtres humains et les animaux. Selon certains de ses informateurs à Iglulik, les mots humains « détruisent » (suraqtut) les animaux (ibid., 7).

23. Notons qu'en inuktitut, l'idée de "faire une marque sur quelque chose, tracer un trait " (taqsaliqpaa) renvoie à la notion d'ombre portée, d'obscurité et de noirceur (taaq-) à partir de laquelle le mot est formé. Selon la pensée inuit, taqsaq est associé tant à la fourrure des animaux, à la couture, à une décoration qu'aux tatouages corporels visibles ou encore à un chemin (Qumaq, 1991, 255). Pour beaucoup d'Inuit, le graphisme (écriture et dessin) se rapproche de la couture et du tatouage, ce dont témoigne la terminologie : aglait : désigne soit les caractères utilisés pour écrire soit les motifs décoratifs que la couturière brode (Nunavik); taaqsituqpaa : il/elle s'est tatoué(e) ; a souligné un mot dans un texte (Nunavik) ; titiqpaa : il/elle trace un repère sur un objet, sur une peau (Nunavik); titirtugaqpuq : il/elle écrit, dessine, trace un trait (sud de l'île de Baffin); titiraujaqsimajuq : écriture, dessin, couture exécutée (nord de l'île de Baffin).

24. Pour une analyse détaillée de ce récit, voir Saladin d'Anglure (2006, 128-132).

25. Selon la définition de T. Qumaq $(1991,376)$, M. Therrien rapporte que le secret dévoilé est perçu comme un objet invisible que l'on extrait de l'eau profonde nuititaq $(2008,276)$.

26. Cependant, l'absence de traces sur le territoire reste associée à la présence des ijirait, ces êtres invisibles qui tentent d'attirer les humains à l'intérieur des terres, dont les traces de pas sont vouées à l'invisibilité (Therrien, 1990, 40).

27. Autrefois, les femmes inuit étaient tatouées à la puberté ou lorsqu'elles se mariaient. Les tatouages étaient tracés sur le visage et le reste du corps, par l'intermédiaire d'une aiguille d'os enduite de suie glissée sous la peau (voir notamment Rasmussen, 1931, 316). Les hommes pouvaient parfois être tatoués sur le visage s'ils avaient commis un crime.

28. Ceci n'est pas sans évoquer l'étude de M. Perrin (2010, 115-128) des dessins des Shipibo (Amazonie péruvienne), selon laquelle la maladie serait due à un dessin corporel mal tracé que personne ne voit excepté le chamane et dont le traitement consiste en un chant (révélé par une vision) qui « prend la forme d'un beau dessin, d'un kikin kene. Il rentre dans le corps du malade. Le chamane recommence plusieurs nuits... Si le dessin ne peut pas pénétrer son corps, le malade meurt » (ibid., 125). Le chant consiste donc en la « réécriture » du dessin dont la substitution par un nouveau redonnera la santé.

29. Ce point sera ultérieurement développé dans un chapitre de ma thèse en préparation (supra, note 3$)$.

30. Selon M. Therrien l'absence de savoirs parfaitement maîtrisés implique la "retenue verbale ", dans un contexte où les procédés linguistiques visent à "lisser les échanges parlés » $(2008,255,257)$. Lors des entrevues, placer la création artistique au centre du discours mettait parfois mal à l'aise les locuteurs. 


\section{RÉSUMÉS}

Cet article consiste en la définition de l'oralité comme principe inhérent de l'art graphique au Nunavut, dans le domaine de la création artistique contemporaine inuit de l'Arctique canadien. Selon une approche ethnolinguistique et anthropologique, il engage une réflexion sur ce que signifient " parler » et « dessiner », en considérant ces deux pratiques comme significatives dans l'élaboration des discours identitaires des sociétés inuit contemporaines. L'analyse des concepts inuit d'« art » (sanannguaq) et de "tracé/trace » (taqsaq) en relation avec la notion de " parole/ mot » (uqausiq), ainsi que l'étude de la cosmogénèse et des témoignages révèlent des corrélations déterminantes quant à une certaine forme de pouvoir assignée tant à la parole/mot qu'au dessin tracé.

This paper consists in defining orality as main principle of graphic art in Nunavut, within the contemporary Inuit artistic domain in the Canadian Arctic. Following an ethnolinguistics and anthropological approach, it engages a reflection about what "talking" means, and about what "drawing" means, considering these both practices significant within the elaboration of identity discourses process among the contemporary Inuit societies. Regarding the analysis of Inuit art concepts (sanannguaq) and "layout/mark" concept (taqsaq) on connection with the notion of "word" (uqausiq), added to the study of cosmogenesis and testimonies reveal significant correlations related to a certain form of power linked between the word and the drawing.

\section{INDEX}

Keywords : Graphic Arts, Nunavut, Inuit, Inuktitut, Anthropology

nomsmotscles Inuit

Thèmes : arts graphiques, anthropologie (Amérique)

Index géographique : Nunavut, Arctique canadien

Mots-clés : Inuktitut 